\title{
Potential antioxidants from crude extracts of roselle seeds and cashew nut shells for biodiesel storage stability improvement
}

Cite as: AIP Conference Proceedings 2129, 020087 (2019); https://doi.org/10.1063/1.5118095

Published Online: 30 July 2019

M. H. Hasan, M. S. M. Adzib, and Z. Ilham

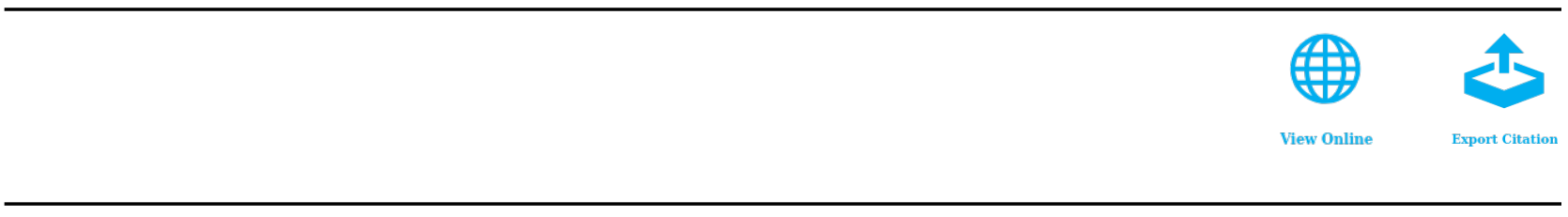

\section{Conference Proceedings}

Get $30 \%$ off all print proceedings!
Enter Promotion Code PDF-30 at checkout
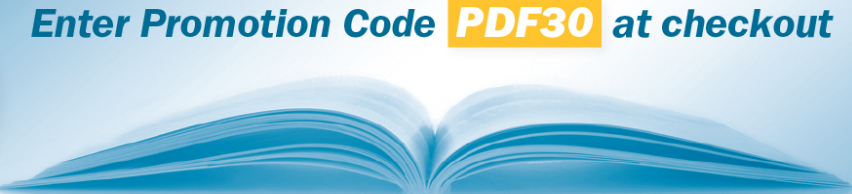


\title{
Potential Antioxidants from Crude Extracts of Roselle Seeds and Cashew Nut Shells for Biodiesel Storage Stability Improvement
}

\author{
M H Hasan ${ }^{1}$, M S M Adzib ${ }^{1}$ and Z Ilham, ${ }^{1, a)}$ \\ ${ }^{I}$ Biomass Energy Lab, Institute of Biological Sciences, Faculty of Science, University of Malaya 50603 Kuala \\ Lumpur, Malaysia. \\ ${ }^{a)}$ Corresponding author: ilham@um.edu.my
}

\begin{abstract}
Biodiesel or fatty acid methyl ester (FAME) is a green alternative fuel that degrades under long-term storage. Antioxidants are utilized in maintaining the oxidation stability of biodiesel by inhibiting the propagation of free radicals resulting in prolonged shelf life. Usage of natural antioxidants from renewable resources is preferred to sustain a green economy and potentially match synthetic antioxidants without being hazardous to the environment. In this study, the potential of antioxidants from crude extracts of Roselle seeds (Hibiscus sabdariffa) and cashew nut shells (Anacardium occidentale) which are agricultural residues that are commonly left unconsumed has been tested for biodiesel storage stability improvement. ABTS antioxidation test on both plant parts showed significant results for all samples regardless of their polarity while $\beta$-Carotene test favored polar samples. Positive correlation of DPPH test and total phenolic content (TPC) test confirmed the antioxidant activity of phenolic compounds in all samples. Total flavonoid content (TFC) test also showed positive results for all samples. GC-MS and LC-MS profiling of both plant parts revealed that all samples contained phenolic compounds with the exception of roselle seeds extracted with hexane (RH), which contained mostly fatty acids. Oxidative stability test using Rancimat test showed that polar samples have superior performance than nonpolar samples whereas 3-pentadecyl phenol showed a poor performance in improving biodiesel storage stability.
\end{abstract}

\section{INTRODUCTION}

Biodiesel is a biomass-derived renewable fuel utilized as an alternative to the conventional petrol and diesel. Biodiesel is produced through transesterification reaction of renewable fats and oils with alcohol to produce long chained fatty acid alkyl esters [1]. One of its advantages is short ignition delay due to higher cetane number which reduces the NOx emission during initial combustion [2]. However, biodiesel is susceptible to oxidation due to the presence of unsaturated fatty acids. These unsaturated fatty acids are prone to degradation, thus reducing biodiesel's shelf life. Oxidative stability of biodiesel can be determined by using Rancimat method by detecting the production of volatile acids in a closed and heated system at $110^{\circ} \mathrm{C}$ [3]. It can be operated under different parameters of interest such as airflow, temperature and oil sample size. It functions by measuring the time needed to maximize the oxidation rate of change of deionized water that was bubbled in volatile acid from the heated sample. The rate of change is known as oxidative stability index (OSI) which correlates with lipid oxidation.

Natural compounds have vast potential as additives as they inhibit and delay oxidation rate of lipids and oils [4] as well as preventing free radical formation [5]. There are several in vitro detection methods that are available for rapid screening and analyzing antioxidant activity. Indirect in vitro methods such as ABTS, DPPH, and FRAP are simple but have limitations. Compounds with different polarity will produce different antioxidant activity depending on the solvent [6]. Extraction of complex natural extract which exhibit a degree of polarity, usually involves solvents with different polarity depending on the compound of interest. Antioxidant activity of extract is tested with different analytical methods to evaluate the capabilities of the extract. Methods that are commonly used to test the antioxidant activity of compounds with different degree of polarity are DPPH, ABTS and $\beta$-Carotene [7]. . In this study, the 
potential of antioxidants from crude extracts of Roselle seeds (Hibiscus sabdariffa) and cashew nut shells (Anacardium occidentale) which are agricultural residues that are commonly left unconsumed has been tested for biodiesel storage stability improvement.

\section{METHODOLOGY}

\section{Extraction of Plants Materials}

Roselle seeds and cashew nut shell samples were dried, grinded into fine powder and extraction was performed by using Soxhlet method. 30 grams of powdered samples were extracted with $350 \mathrm{~mL}$ solvent of hexane and methanol for 8 hours at $70^{\circ} \mathrm{C}$. The solvent was then evaporated by using rotary evaporator at $60^{\circ} \mathrm{C}$. Yield of crude extract were calculated by using the formula of: [extracted dried mass (g) / dried crude mass (g)] X 100.

\section{DPPH free radical-scavenging activity}

The DPPH free radical scavenging activity was determined according to the method of Mohd-Esa et al. (2010) with slight modification. $1 \mathrm{~mL}$ of freshly prepared $0.1 \mathrm{M} \mathrm{DPPH}$ in methanol was added into each cuvette containing samples in a range of five different concentrations $(\mathrm{mg} / \mathrm{mL})$ and tested after 5 minutes. Antioxidant activity was calculated by using formula: (absorbance of control - absorbance of sample /absorbance of control) X 100.

\section{$\beta$-Carotene bleaching assay}

This method was prepared according to the method of Barros et al. (2008) with slight modification. $2 \mathrm{mg}$ of $\beta$ Carotene was dissolved in $10 \mathrm{~mL}$ of chloroform and then $2 \mathrm{~mL}$ of this solution was pipetted into a $100 \mathrm{~mL}$ roundbottom flask. The chloroform was removed by using rotary evaporator at $40^{\circ} \mathrm{C}$ and $100 \mathrm{~mL}$ of methanol was added, followed by $20 \mu \mathrm{L}$ linoleic acid and $200 \mu \mathrm{L}$ of Tween 20 emulsifier. Briefly, each sample was diluted until 5 consequence concentration. For every $100 \mu \mathrm{L}$ samples, $1 \mathrm{~mL}$ of aliquot was used and was measured at $470 \mathrm{~nm}$ by using UV-visible spectrometer at 0 minute as initial reading. Then, the samples were incubated at $50^{\circ} \mathrm{C}$ in water bath and final reading was taken at 120 minutes. A blank, devoid of $\beta$-Carotene, was prepared for background subtraction. Antioxidant activity was calculated by using formula of: (Absorbance reading after 120 minutes at 0 minute) $X 100$.

\section{ABTS radical scavenging activity}

The ABTS radical cation was produced using Miller et al. (1993) method with slight modification. A solution of ABTS $(10 \mathrm{mg})$ and potassium peroxodisulfate $(2.9 \mathrm{mg})$ was diluted with $0.01 \mathrm{M} \mathrm{pH} 7.4$ phosphate buffer $(10 \mathrm{~mL})$. The mixture was protected from light and stored at room temperature for 12-16 hours. ABTS formation was confirmed by absorbance reading of $734 \mathrm{~nm}$. The ABTS solution was diluted with methanol to an absorbance of $0.80( \pm 0.05)$ at $734 \mathrm{~nm}$. For the assays, five concentration of samples were mixed with $1 \mathrm{~mL}$ of ABTS solution. Reduction of absorbance was measured at $734 \mathrm{~nm}$ after 5 minutes. Antioxidant activity was calculated by using formula: (absorbance of control - absorbance of sample labsorbance of control) X 100.

\section{Total Phenolic Content Determination}

Five different concentrations of each sample were determined according to the method of Mohd-Esa et al. (2010). Each sample was added with $1 \mathrm{~mL}$ of $0.5 \mathrm{M}$ Folin-Ciocalteu reagent and shaken for 5 minutes. Then, $1 \mathrm{~mL}$ of $75 \mathrm{~g} / \mathrm{L}$ sodium bicarbonate was added and the mixture was shaken again for 30 seconds. After 5 minutes, the absorbance was measured with UV/Vis spectrophotometer at $760 \mathrm{~nm}$. The phenolic content was determined by plotting the gallic acid calibration curve. 


\section{Total Flavonoid Content Determination}

$100 \mathrm{~mL}$ of $10 \%$ aluminum chloride with $1 \mathrm{M}$ of potassium acetate was prepared. Then, $3.8 \mathrm{~mL}$ of methanol was added at room temperature for 40 minutes. Sample was mixed with the above aliquot and analyzed using UV-visible spectrophotometer at $415 \mathrm{~nm}$. The flavonoids were determined by plotting the quercetin calibration curve.

\section{Gas Chromatography Mass Spectrometry (GC-MS)}

GC-MS analysis was done using Agilent 7000 GC-QQQ gas chromatography coupled to ion trap mass spectrometer. The column was HP-5MS with $5 \%$ phenyl methyl polysiloxane. The oven temperature was programmed to have the initial temperature of $70^{\circ} \mathrm{C}$ for 0 minute and increased gradually with $10^{\circ} \mathrm{C} / \mathrm{min}$ up to $300^{\circ} \mathrm{C}$ and was held for 6 minutes. Carrier gas was Helium at $1 \mathrm{~mL} / \mathrm{min}$ in constant flow mode with splitless injection temperature of $250^{\circ} \mathrm{C}$, auxiliary temperature of $280^{\circ} \mathrm{C}$ and threshold at $150^{\circ} \mathrm{C}$.

\section{Liquid Chromatography Mass Spectrometry (LC-MS)}

The samples were determined by LC-MS/MS method using AB Sciex 3200Q Trap LC-MS/MS with Perkin Elmer FX 15 uHPLC system. Agilent Zorbax C18, $150 \mathrm{~mm}$ x $4.6 \mathrm{~mm}$ x $5 \mu \mathrm{m}$ was used as the column. Two types of buffer were used; Solvent A: water with $0.1 \%$ formic acid and $5 \mathrm{mM}$ ammonium formate and Solvent B: Acetonitrile with $0.1 \%$ formic acid and $5 \mathrm{mM}$ ammonium formate. For the MS settings, the voltage IS and source temperature were set at $5500 \mathrm{~V}$ with temperature of $500^{\circ} \mathrm{C}$. The gradient run program was set as the following: $10 \%$ $\mathrm{B}$ to $90 \% \mathrm{~B}$ from 0.01 minutes to 8 minutes. It was held for 3 minutes and back to $10 \% \mathrm{~B}$ in 0.1 minutes and reequilibrated for 5 minutes. The sample was diluted in $2 \mathrm{~mL}$ solvent and filtered with nylon $0.22 \mu \mathrm{M}$. Analysis was carried out by injecting $20 \mu \mathrm{L}$ of diluted sample solution.

\section{Oxidative Stability Test}

The usage of natural plants extracted with different solvents were tested by using the Metrohm Rancimat (Metrohm 873) following the European Standard of EN 14214 and EN 14112. Synthetic antioxidants of BHA, 3pentadecyl phenol (analytical grade) and Trolox ${ }^{\circledR}$ were also tested with the same procedure as the positive control. Each sample and positive control were tested with palm oil biodiesel (fatty acid methyl esters) from Weschem Technologies Sdn. Bhd. with three range of weight/weight percentage (w/w \%) at temperature of $110^{\circ} \mathrm{C}$ with gas flow rate of $10 \mathrm{~L} / \mathrm{h}$.

\section{RESULTS AND DISCUSSIONS}

\section{Yield of Crude Extract}

The yield of crude extract for methanol extract of cashew nut shells (CM), hexane extract of cashew nut shells $(\mathrm{CH})$, methanol extract of Roselle seeds (RM) and hexane extract of Roselle seeds (RH) were tabulated in Table 1.

TABLE 1. Yield of crude extract.

\begin{tabular}{lcccc}
\hline & CM & RM & CH & RH \\
\hline Dried weight (g) & 11.16 & 5.21 & 10.17 & 4.31 \\
Percentage (\%) & 37.20 & 17.37 & 33.90 & 14.37 \\
\hline
\end{tabular}




\section{DPPH Free Radical Scavenging Activity Assay}

Statistical analysis showed that all the extracts were significantly different $(\mathrm{p}<0.05)$ and the $\mathrm{IC}_{50}$ order was Trolox ${ }^{\circledR}>$ BHA $>\mathrm{CM}>\mathrm{RM}$. For $\mathrm{CH}$ and $\mathrm{RH}$, the $\mathrm{IC}_{50}$ was not achieved (Table 2). The lower inhibition percentage of hexane extracts when compared to methanol extracts could be due to the difference in polarity of the solvent used during the assay testing. DPPH scavenging assay favored extraction with polar solvent instead of nonpolar solvent which corresponded with a previous study [8]. This is also demonstrated by hexane's DPPH radical scavenging activity which was the lowest among ethanol, methanol and acetone. Furthermore, methanol with polarity index of 5.1 exhibited the highest total phenolic content compared to the other solvents [9]. Therefore, solvent with intermediate polarity is preferred over extremely polar or nonpolar solvents [10].

\section{ß-Carotene Bleaching Assay}

The presence of antioxidants prevented free radical formation which are formed from linoleic acid that attack the highly unsaturated $\beta$-Carotene [11]. The antioxidant activity of samples was presented in Table 2 . The order of $\beta$ Carotene free radical scavenging activities of the extracts was Trolox ${ }^{\circledR}>\mathrm{CH}>\mathrm{RH}(\mathrm{p}<0.05)$. For $\mathrm{CM}$ and $\mathrm{RM}$, there were no linear correlations of antioxidant activity. The inconsistency of absorbance reading of polar extracted compounds such as CM, RM and positive control (BHA) could be explained by the polar paradox theory [12]. Nonpolar lipophilic antioxidants hindered the transport of free radicals across the droplet membrane as the nonpolar antioxidants formed a protective layer around the oil droplet. However in $\beta$-Carotene emulsion system, nonpolar antioxidants were more effective than polar antioxidants which is more effective in bulk oil [13] and lipophilic antioxidants are more active in polar mediums, the mechanism of polar paradox [14]. When tested with $\beta$-Carotene assay, polar compound such as ascorbic acid exhibited weak antioxidant activity but exhibited strong antioxidant activity when tested with DPPH assay [15]. This indicated that $\beta$-Carotene assay is unsuitable in determining the antioxidant activity of polar antioxidants. Thus, polar extract showed a similar trend as polar paradox as none of the polar extracted samples showed a consistent correlation between each concentration.

\section{ABTS Radical Scavenging Activity Assay}

The ABTS radical scavenging activity of all samples were tabulated in Table 2 with $\mathrm{p}<0.05$ excluding RM and $\mathrm{RH}$. The $\mathrm{IC}_{50}$ order of ABTS radical scavenging activities of the extracts was Trolox ${ }^{\circledR}>\mathrm{BHA}>\mathrm{CM}$ and $\mathrm{CH}>\mathrm{RM}$ and $\mathrm{RH}$. The $\mathrm{IC}_{50}$ of $\mathrm{CM}$ and $\mathrm{RM}$ means were significantly different. $\mathrm{RH}$ exhibited weakest scavenging activity among all other tested samples. In ABTS scavenging activity, it should be noted that both $\mathrm{CH}$ and $\mathrm{RH}$ achieved $50 \%$ of inhibition but this were not demonstrated in DPPH assay. This was due to the characteristics of ABTS that favored both polar and nonpolar extracts. As a result, ABTS was suitable to determine both hydrophilic and lipophilic antioxidants [16-17]. It can also be utilized to evaluate plant extracts of both organic and aqueous media [18].

TABLE 2. IC50 scavenging activity as in $\mathrm{mg} / \mathrm{mL}$.

\begin{tabular}{|c|c|c|c|c|}
\hline & CM & $\mathbf{R M}$ & $\mathbf{C H}$ & RH \\
\hline DPPH & $5.48 \pm 0.31$ & $7.58 \pm 0.15$ & $\begin{array}{l}\mathrm{IC}_{50} \text { not } \\
\text { achieved }\end{array}$ & $\begin{array}{l}\mathrm{IC}_{50} \text { not } \\
\text { achieved }\end{array}$ \\
\hline$\beta$-Carotene & No correlation ${ }^{1}$ & No correlation ${ }^{1}$ & $8.01 \pm 0.24$ & $21.12 \pm 0.16$ \\
\hline ABTS & $5.20 \pm 0.41$ & $12.61 \pm 0.11$ & $5.23 \pm 0.37$ & $12.884 \pm 0.28$ \\
\hline
\end{tabular}

In determining the antioxidant activity of a sample, it is crucial to confirm the completion of reaction before measuring the absorbance as there are three types of kinetic behavior, namely rapid kinetic behavior (less than 1 minute), intermediate kinetic behavior (5 to 30 minutes) and slow kinetic behavior (1 to 6 hours) [14]. Therefore, 
analyzing the data in certain interval will not be accurate as some of the antioxidants are still undergoing reaction. Thus, it is recommended to test the antioxidants during the steady state where the absorbance is already constant.

\section{Total Phenolic Content (TPC) Determination}

Overall, CM exhibited the highest phenolic content. Data were tabulated in Table 3 and the result was in the order of $\mathrm{CM}>\mathrm{RM}$ and $\mathrm{CH}>\mathrm{RH}$ with respect to gallic acid concentration $(\mathrm{p}<0.05)$ excluding $\mathrm{RM}$ and $\mathrm{CH}$. The use of Folin-Ciocalteau reagent was not specific for phenols as other compounds such as ascorbic acid could interfere with the reagent [15]. Hence, confirmation by chromatography need to be performed.

\section{Total Flavonoid Content (TFC) Determination}

The total flavonoid content for all four extracts were presented in Table 3 with the highest content exhibited by RM while the lowest was RH. It should be noted that the flavonoid content of RM was about 4 times higher than RH while the rest were in the range of 4 to $5 \mathrm{mg} / \mathrm{g}$. The result was in the order of $\mathrm{RM}$ and $\mathrm{CM}>\mathrm{CH}>\mathrm{RH}(\mathrm{p}<0.05)$ excluding RM and CM.

TABLE 3. Total phenolic and flavonoid contents in $\mathrm{mg} / \mathrm{g}$ at concentration of $14.57 \mathrm{mg} / \mathrm{ml}$.

\begin{tabular}{ccccc}
\hline & CM & RM & CH & RH \\
\hline TPC(mg/g GAE) & $3.776 \pm 0.13$ & $3.194 \pm 0.11$ & $2.806 \pm 0.17$ & $1.043 \pm 0.21$ \\
TFC(mg/g QAE) & $4.538 \pm 0.34$ & $4.814 \pm 0.48$ & $4.147 \pm 0.47$ & $1.143 \pm 0.33$ \\
\hline
\end{tabular}

\section{Gas Chromatography Mass Spectrometry (GC-MS)}

From the GC-MS profiling, 4 compounds of phenols were identified from $\mathrm{CH}$ and none from RH (Table 4). Commonly, 2-methy phenol is known as o-cresol [19], which consisted of a methyl group attached at the orthoposition of the benzene ring [20]. Other compounds that were identified in this extract were 2-methyl-1,4benzenediol and 4,5-methyl-1,3-benzenediol, although there was not much information from previous studies concerning these two compounds. 3-pentadecyl phenol or alkyl phenolic oil, is part of the cardanol group and was present in the spongy mesocarp of the cashew nut shells [21-22]. High amounts of cardanol were a product of anacardic acid decarboxylation at high temperature [23]. However, high temperature will accelerate the reaction and produce unwanted side chain [24]. Thus, long extraction time and the application of high temperature need to be carefully considered to accelerate the conversion. For roselle seeds, the compound identified were $n$-hexadecanoic acid and 9, 12-octadecadienoic acid (Table 4).

TABLE 4. GCMS analysis of cashew nut shells extracted with hexane (CH).

\begin{tabular}{cccc} 
Extract & Retention Time & Area (\%) & Name of Compound \\
\hline $\mathrm{CH}$ & 16.83 & 2.59 & 3-pentadecyl phenol \\
& 18.70 & 7.80 & 3-pentadecyl phenol \\
& 28.87 & 2-methyl phenol \\
& 21.96 & 8.6 & 2-methyl phenol \\
& 18.39 & 2-methyl-1,4-benzenediol \\
& 22.94 & 8.76 & 4,5-methyl-1,3-benzenediol \\
\hline
\end{tabular}




\section{Liquid Chromatography Mass Spectrometry (LC-MS)}

All the identified compounds were polar as exhibited by the presence of at least one hydroxyl group connected to the aromatic benzene ring (Table 5). Naringenin and caffeic acid were highly present in cashew leaves and nuts [25]. In addition, caffeic acid is the most concentrated phenolic compound identified in cashew apple juice [26] while ellagic acid is a flavonoid compound which can be found in the leaf of a roselle plant [27-28].

TABLE 5. LCMS analysis of cashew nut shells (CM) and roselle seeds (RM) extracted with methanol.

\begin{tabular}{ccc}
\hline Extracts & Retention Time & Name of Compound \\
\hline CM & 2.87 & Naringenin \\
& 3.38 & Naringin \\
& 3.96 & Naringin \\
RM & 4.21 & Ellagic acid \\
\hline
\end{tabular}

\section{Oxidative Stability Test}

Oxidative stability test was performed using Rancimat machine following a European standard EN14214 for biodiesel which set the induction period for 6 hours as the lower limit and EN 14112 standard method to determine the oxidation stability for fatty acid methyl esters using accelerated oxidation test. The induction period of samples and positive control were shown in Fig. 1.

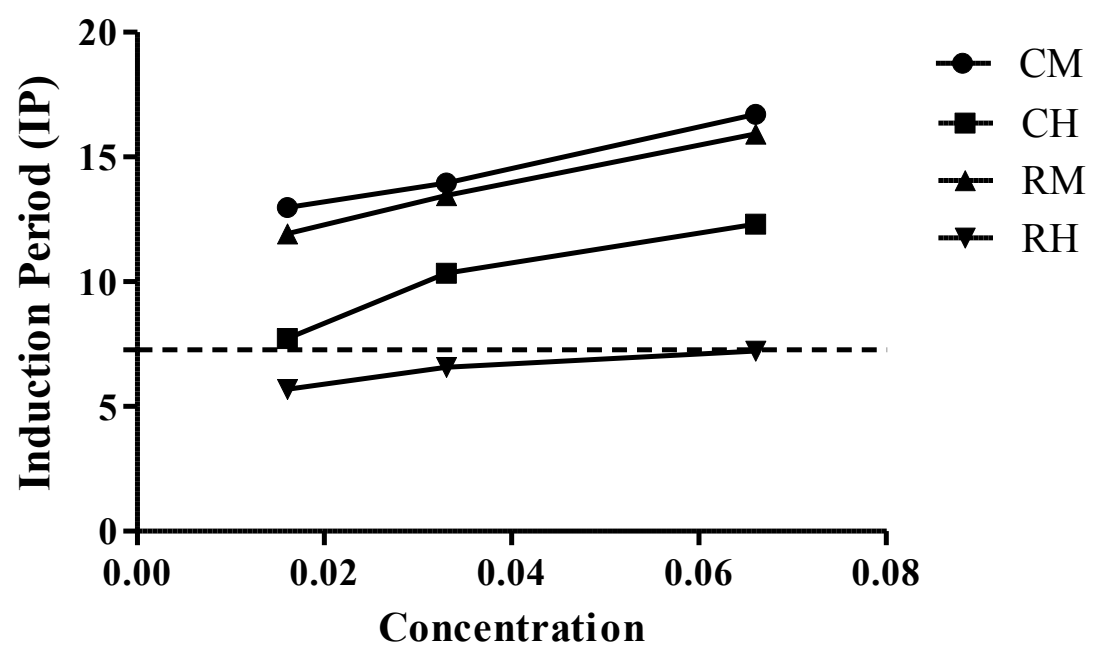

FIGURE 1. Induction period of samples and positive controls.

Biodiesel without the addition of antioxidant served as a control for this test and exhibited an induction period of 7.27 hours (dotted line across Y-axis). This was higher than the induction period of biodiesel with the addition of 3pentadecyl phenol (3DP) as antioxidant. The reactive free hydroxyl group could be attached to a variety of functional group through series of reaction such as nucleophilic aromatic or aromatic substitution and condensation reaction [29]. Then, intermediate compounds will be attacked by the nucleophilic cardanols and polymerized into a new compound [30]. All of these suggested the attack on the unsaturated chain by the nucleophilic aromatic 3pentadecyl phenol, thus diminishing the biodiesel's shelf life.

Another cause was the presence of high degree of unsaturated meta-substituent attached to the aromatic ring of 3DP. Meta-alkylphenols attached to the benzene ring varied in degree of unsaturation [2]. Free radicals attacked the unsaturated alkyl chain and underwent propagation reaction to form another free radical chain. This was the same for RH which showed lower induction period than control due to RH's high content of unsaturated fatty acid $(9,12-$ octadecdienoic acid) rather than phenolic compound. The unsaturated compound was prone to oxidation which led to lower induction period compared to control. The nonpolar $\mathrm{CH}$ showed an increasing trend of induction period. This proved that nonpolar extract could be utilized as an antioxidant additive in biodiesel. 
However, the availability of multiple compounds did not equate a higher induction period. For example, the induction period of $\mathrm{CH}$ was lower compared to RM although there were 4 types of phenolic compounds in $\mathrm{CH}$. In contrast, RM only had a single phenolic compound: ellagic acid. This was consistent with the earlier argument that all the compounds in these extracts did not act alone to combat the free radical as they are not high in purity. it is rather a combination of several compounds of antioxidant that work together in one mechanism to fight the free radicals.

\section{CONCLUSIONS}

The solvent type, temperature and extraction method are fundamental aspects in extraction due to their significance in multiple tests that were conducted in this study. Solvent selection for extraction is mainly influenced by the compound of interest. The solvent polarity also affected the antioxidant assay. Generally, plant consists of many compounds that have different polarity. Thus, the usage of different solvents for extraction was practical in this study as extraction of compounds such as flavonoids were enhanced using polar solvents instead of nonpolar. Therefore, it can be concluded that difference in solvent polarity affected the extraction yield.

The oxidative stability test showed that polar extracts exhibited superior induction period when compared to nonpolar extracts. On the other hand, Ellagic acid, which was the single compound found in RM, exhibited almost the same induction period as CM, which contained four compounds. By excluding the polarity effect as both were methanol extracts, it can be concluded that ellagic acid had a higher antioxidant capacity in biodiesel. In extraction, temperature is crucial in retaining the compound in their natural state as some compounds might be degraded by the temperature and undergo minor changes in their side chain. It is recommended to use low temperature extraction methods with shorter extraction time as high temperature might break or alter the structure of the compound and cause the compound to be fragmented into smaller nonfunctional structure. For future works, it is recommended to separate the pure methanol extract into several partitions by using other solvent with differing polarity such as hexane and chloroform. Hence, by testing the content of extract in the partitioned solvent, identification of the compounds responsible for the antioxidant activity can also be performed. Lastly, in oxidative stability test, it is best to extrapolate a graph by using at least 5 different antioxidant concentrations as the data can be further manipulated for further use such as determining the optimum antioxidant concentration.

\section{ACKNOWLEDGMENTS}

This work was supported research grants by University of Malaya (GPF016B-2018 and PO026-2014A), which the authors highly acknowledged.

\section{REFERENCES}

1. S. Howell, "Animal and Vegetable Fats and Oils-Determination of Oxidative Stability (Accelerated Oxidation Test" (International Organization for Standards, 1996).

2. F. H. A. Rodrigues, J. Feitosa, N.M. Ricardo, F.C.F.D. França, and J.O.B. Carioca, J. Braz. Chem. Soc. 17, 265271 (2006).

3. K. Ryu, Bioresour. Technol. 101, S78-S82 (2010).

4. P. R. Venskutonis, D. Gruzdiene, D. Tirzite and G. Tirzitis, Acta Hortic 677, 99-107 (2005).

5. V. Katalinic, M. Milos, T. Kulisic and M. Jukic, Food Chem. 94, 550-557 (2006).

6. K. Pyrzynska and A. Pękal, Anal Methods 5, 4288-4295 (2013).

7. M. Á. P. Lage, M. A. M. García, J. A. V. Álvarez, Y. Anders and T. P. Curran, Food Res. Int. 53, 836-846 (2013).

8. H. K. Makari and R. Patil, The Internet Journal of Aesthetic and Antiaging Medicine 1, 1-10 (2008).

9. L. Y. Ng, Y. K. Ang, H. E. Khoo and H. S. Yim, Research Journal of Phytochemistry 6 (2012)

10. J. Pokorný and J. Korczak, "Antioxidants in Food: Practical Application" (Woodhead Publishing Limited, Cambridge, 2001).

11. L. Barros, S. Falcão, P. Baptista, C. Freire, M. Vilas-Boas and I. C. Ferreira, Food Chem. 111, 61-66 (2008)

12. E. N. Frankel, S. W. Huang, R. Aeschbach and E. Prior, J. Agric. Food Chem. 44, 131-135 (1996)

13. H. S. Yim, F. Y. Chye, C. T. Tan, Y. C. Ng and C. W. Ho, Malays. J. Nutr. 16, 281-291 (2010).

14. W. Brand-Williams, M. E. Cuvelier and C. L. W. T. Berset, LWT- Food Sci Technol 28, 25-30 (1995). 
15. P. Siramon and Y. Ohtani, J Wood Sci 53, 498-504 (2007).

16. R. L. Prior, X. Wu and K. Schaich, J. Agric. Food Chem. 53, 4290-4302 (2005).

17. A. L. Dawidowicz and M. Olszowy, Eur. Food Res. Technol. 236, 1099-1105 (2013).

18. M. B. Arnao, A. Cano and M. Acosta, Food Chem. 73, 239-244 (2001).

19. M. Weber and M. Weber, "Phenolic Resins : A Century of Progress" (Springer, Berlin, Heidelberg, 2010).

20. S. M. Mukherji, S. P. Singh and R. P. Kapoor, "Organic Chemistry" (2 ${ }^{\text {nd }}$ edn., New Age International (P) Ltd Publishers, India, 2014).

21. O. A. Attanasi, G. Mele, P. Filippone, S. E. Mazzetto and G. Vasapollo, Arkivoc 8, 69-84 (2009).

22. T. D. J. A. D. S. Andrade, B. Q. Araújo, A. M. D. G. L. Citó, J. da Silva, J. Saffi, M. F. Richter and A. D. B. F. Ferraz, Food Chem. 126, 1044-1048 (2011).

23. R. Paramashivappa, P. P. Kumar, P. J. Vithayathil and A. S. Rao, J. Agric. Food Chem. 49, 2548-2551(2001).

24. A. C. Kumoro, M. Hasan and H. Singh, Scienceasia 35, 306-309 (2009).

25. A. E. Nugroho, A. Malik and S. Pramono, Int Food Res J 20, 299-305 (2013).

26. A. D. O. U. Marc, K. D. Ange, T. F. Achille and A. N. Georges, J. Appl. Biosci. 49, 3331-3338 (2012).

27. I. Da-Costa-Rocha, B. Bonnlaender, H. Sievers, I. Pischel and M. Heinrich, Food Chem. 165, 424-443 (2014).

28. E. H. Kang, T. Y. Kown, G. T. Oh, W. F. Park, S. I. Park, S. K. Park and Y. I. Lee, Antiviral Res. 72, $100-106$ (2006)

29. B. Lochab, I. K. Varma and J. Bijwea, Advances in Materials Physics and Chemistry 2, 221 (2013).

30. D. S. Souza, R. D. S. Lopes, D. P. de Lima, K. T. D. Oliveira and A. Beatriz, Orbital: Electron. J. Chem. 4, 5556 (2012). 